\title{
Regular submodules of torsion modules over a discrete valuation domain
}

\author{
Pudji Astuti ${ }^{1}$ \\ Departemen Matematika \\ Institut Teknologi Bandung \\ Bandung 40132 \\ Indonesia
}

\author{
Harald K. Wimmer \\ Mathematisches Institut \\ Universität Würzburg \\ D-97074 Würzburg \\ Germany
}

April 21, 2022

\footnotetext{
${ }^{1}$ The research of the first author was supported by Deutscher Akademischer Austauschdienst under Award No. A/98/25636.
} 


\begin{abstract}
A submodule $W$ of a p-primary module $M$ of bounded order is known to be regular if $W$ and $M$ have simultaneous bases. In this paper we derive necessary and sufficient conditions for regularity of a submodule.
\end{abstract}

Mathematical Subject Classifications (2000): 13C12, 20K10, 20K25

Keywords: regular submodules, modules over discrete valuation domains, abelian p-groups, simultaneous bases 


\section{Introduction}

Let $R$ be a discrete valuation domain with maximal ideal $R p$, and let $M$ be a torsion module over $R$ and $W$ be a submodule of $M$. The submodule $W$ is called regular [5, p.65], [6, p.102] if

$$
p^{n} W \cap p^{n+r} M=p^{n}\left(W \cap p^{r} M\right)
$$

holds for all $n \geq 0, r \geq 0$. The regularity condition (1.1) was introduced by Vilenkin [6] in his study of decompositions of topological p-groups. Kaplanski [5] showed that for a module $M$ of bounded order (1.1) is necessary and suffifient for the existence of simultaneous bases of $W$ and $M$. In this paper we shall identify two conditions which are equivalent to (1.1). One is related to a theorem of Baer [4, p.4] on the decomposition of elements in abelian p-groups, the other one was introduced by Ferrer, F. Puerta and X. Puerta [2] to characterize marked invariant subspaces of a linear operator.

Notation and definitions: The letters $\mathcal{U}, \mathcal{V}, \mathcal{X}, \ldots$, will always denote subsets of $M$. Let $\langle\mathcal{X}\rangle$ be the submodule spanned by $\mathcal{X}$. We shall use the letters $u, v, x, \ldots$, for elements of the module $M$, and $\alpha, \beta, \mu, \ldots$, will be elements of the ring $R$. Using the terminology for abelian $p$-groups in [3, p.4] we say that $x \in M$ has exponent $k$, and we write $\mathrm{e}(x)=k$, if $k$ is the smallest nonnegative integer such that $p^{k} x=0$. An element $x \in M$ is said to have (finite) height $s$ if $x \in p^{s} M$ and $x \notin p^{s+1} M$, and $x$ has infinite height, if $x \in p^{s} M$ for all $s \geq 0$. We write $\mathrm{h}(x)$ for the height of $x$. If $x \in W$ then $\mathrm{h}_{W}(x)$ will denote the height of $x$ with respect to $W$. Note that $\mathrm{e}(0)=0$ and $\mathrm{h}(0)=\infty$. Let $R^{*}$ be the group of units of $R$. If $\alpha \in R$ is nonzero and $\alpha=p^{s} \gamma, \gamma \in R^{*}$, then we set $\mathrm{h}(\alpha)=s$. We put $\mathrm{h}(\alpha)=\infty$ if $\alpha=0$. We call $x \in M$ an $\left(s, k ; s_{1}\right)$-element if $x \neq 0$ and

$$
\mathrm{h}(x)=s, \mathrm{e}(x)=k, \mathrm{~h}\left(p^{k-1} x\right)=(k-1)+s_{1} .
$$

In accordance with a definition of Baer [1] we say that an element $x$ is regular if $\mathrm{h}(x)=\infty$ or if $\mathrm{h}(x)$ is finite and

$$
\mathrm{h}\left(p^{j} x\right)=j+\mathrm{h}(x), j=1, \ldots, \mathrm{e}(x)-1 .
$$

The two concepts of regularity introduced above are consistent. We shall see in Lemma 3.2 that a finite height element $x \in M$ is regular if and only if $\langle x\rangle$ is a regular submodule of $M$. and

For $s \geq 0, k \geq 0$ we define the submodules $M\left[p^{k}\right]=\left\{x \in M \mid p^{k} x=0\right\}$

$$
M_{k}^{s}=p^{s} M \cap M\left[p^{k}\right] .
$$


Then

$$
M_{k}^{s}=\{x \in M \mid \mathrm{e}(x) \leq k, \mathrm{~h}(x) \geq s\} .
$$

In particular $M_{0}^{s}=0$.

Our main result will be the following.

Theorem 1.1. Let $M$ be a torsion module over a discrete valuation domain and let $W$ be a submodule of $M$. The following conditions are equivalent.

(K) $W$ is regular, i.e. if $n \geq 0, r \geq 0$ then

$$
p^{n} W \cap p^{n+r} M=p^{n}\left(W \cap p^{r} M\right) .
$$

(B) If $x \in W$ is nonzero then $x$ can be decomposed as

$$
x=y_{k_{1}}^{s_{1}}+\cdots+y_{k_{m}}^{s_{m}}
$$

such that

$$
y_{k_{i}}^{s_{i}} \in W \text { is regular, } i=1, \ldots, m
$$

and

$$
\mathrm{h}\left(y_{k_{i}}^{s_{i}}\right)=s_{i}, \mathrm{e}\left(y_{k_{i}}^{s_{i}}\right)=k_{i}
$$

and

$$
k_{1}>\cdots>k_{m}>0 \text { and } s_{1}>\cdots>s_{m} .
$$

(FPP) If $s \geq 0, k \geq 1$, then

$$
\left(W \cap M_{k}^{s+1}\right)+\left(W \cap M_{k-1}^{s}\right)=W \cap\left(M_{k}^{s+1}+M_{k-1}^{s}\right) .
$$

By a result of Baer [4, p.4, Lemma 65.4] condition (B) is satisfied for $W=M$. Hence (B) singles out those submodules $W$ where each element $x \in W$ allows a decomposition (1.5) such that the summands $y_{k_{i}}^{s_{i}}$ can be chosen from $W$ itself. With regard to condition (FPP) we observe that the inclusion

$$
\left(W \cap M_{k}^{s+1}\right)+\left(W \cap M_{k-1}^{s}\right) \subseteq W \cap\left(M_{k}^{s+1}+M_{k-1}^{s}\right)
$$

holds for all submodules $W$.

The proof of the theorem will be split into two parts. In Section 3 we show that $(\mathrm{B})$ and $(\mathrm{K})$ are equivalent and in Section 4 we prove the equivalence of (B) and (FPP). 


\section{Decomposition of elements}

We introduce a condition which will be the link between $(\mathrm{B})$ and $(\mathrm{K})$ on one hand and between (B) and (FPP) on the other. For a submodule $W$ we define condition $(\mathrm{H})$ as follows.

(H) If $x \in W$ is an $\left(s, k ; s_{1}\right)$-element then $x$ can be decomposed as

$$
x=y_{k}^{s_{1}}+z, y_{k}^{s_{1}} \in W, z \in W
$$

such that

$$
\mathrm{h}\left(y_{k}^{s_{1}}\right)=s_{1}, \mathrm{e}\left(y_{k}^{s_{1}}\right)=k \text {, and } \mathrm{h}(z)=s, \mathrm{e}(z)<k
$$

The following technical lemma will be useful in several instances. It implies that the element $y_{k}^{s_{1}}$ in (2.1) is regular .

Lemma 2.1. Let $x \in M$ be an $\left(s, k ; s_{1}\right)$-element. Assume

$$
x=y+z, z \in M_{k-1}^{s} .
$$

Then $y \neq 0$, $\mathrm{e}(y)=k$, and

$$
s \leq \mathrm{h}(y) \leq s_{1}
$$

The element $y$ is regular if and only if $\mathrm{h}(y)=s_{1}$. If $x$ is regular then (2.3) implies $\mathrm{h}(y)=s$.

Proof: From (2.3) follows $p^{k-1} y=p^{k-1} x \neq 0$, and $\mathrm{e}(y)=k$. Therefore

$$
(k-1)+\mathrm{h}(y) \leq \mathrm{h}\left(p^{k-1} y\right)=\mathrm{h}\left(p^{k-1} x\right)=(k-1)+s_{1},
$$

which yields $\mathrm{h}(y) \leq s_{1}$. It is obvious from (2.5) that we have $\mathrm{h}(y)=s_{1}$ if and only if

$$
\mathrm{h}\left(p^{k-1} y\right)=(k-1)+\mathrm{h}(y)
$$

i.e., if and only if $y$ is regular. If $x$ is regular then $s_{1}=s$ and (2.4) yields $\mathrm{h}(y)=s$.

Lemma 2.2. For a submodule $W$ the conditions $(\mathrm{B})$ and $(\mathrm{H})$ are equivalent.

Proof: There is nothing to prove if $x$ is regular. Thus, in the following we assume that $x$ is a non-regular element of $W$ with $\mathrm{h}(x)=s$ and $\mathrm{e}(x)=k$. In that case we have $k>1, s_{1}>s$, and $\mathrm{h}\left(p^{k-1} x\right)=(k-1)+s_{1}$.

$(\mathbf{B}) \Rightarrow(\mathbf{H})$ Let $x$ be given as in (1.5), with $m \geq 2$. Put $z=y_{k_{2}}^{s_{2}}+\cdots+y_{k_{m}}^{s_{m}}$. 
Then (1.6) implies $\mathrm{e}(z) \leq k_{2}<k$ and $\mathrm{h}(z)=s_{m}=s$. Hence the decomposition $x=y_{k_{1}}^{s_{1}}+z$ is of type $(\mathrm{H})$.

$(\mathbf{H}) \Rightarrow(\mathbf{B})$ Let $x$ be an $\left(s, k ; s_{1}\right)$-element of $W$ and assume that $x$ is decomposed according to $(\mathrm{H})$ as

$$
x=y_{k}^{s_{1}}+z
$$

such that (2.2) holds. We know from Lemma 2.1 that $y_{k}^{s_{1}}$ is regular. Consider $x$ with $s_{1}>s, k>1$. Assume as an induction hypothesis that condition $(\mathrm{H})$ ensures a decomposition of type (B) for all $w \in W$ with $\mathrm{e}(w)<k$. Thus we have

$$
z=z_{l_{2}}^{t_{2}}+\cdots+z_{l_{m}}^{t_{m}}, m \geq 2,
$$

with properties in accordance with $(\mathrm{B})$. Thus $\mathrm{h}\left(p^{l_{2}-1} z\right)=\left(l_{2}-1\right)+t_{2}, t_{2} \geq s$, and $t_{2}>\cdots>t_{m}=s=\mathrm{h}(z)$, and $k>\mathrm{e}(z)=l_{2}>\cdots>l_{m}>0$. If $s_{1}>t_{2}$ then we already have the desired decomposition. Now suppose $t_{2} \geq s_{1}$. Let $j$ be such that

$$
t_{2}>\cdots t_{j} \geq s_{1}>t_{j+1}
$$

Note that $t_{m} \geq s_{1}$ can not occur because of $t_{m}=s$ and $s_{1}>s$. Set

$$
v=y_{k}^{s_{1}}+\left(z_{l_{2}}^{t_{2}}+\cdots+z_{l_{j}}^{t_{j}}\right) .
$$

Then $k>l_{2}$ yields e $(v)=k$. Since $y_{k}^{s_{1}}$ is regular we see that $p^{k-1} v=p^{k-1} y_{k}^{s_{1}}$ implies $(k-1)+s_{1}=\mathrm{h}\left(p^{k-1} v\right)$. Hence $\mathrm{h}(v) \leq s_{1}$. On the other hand it follows from (2.7) that $\mathrm{h}(v) \geq s_{1}$. Therefore $\mathrm{h}(v)=s_{1}$, and $v$ is regular. If we rewrite (2.6) in the form

$$
x=v+z_{l_{j+1}}^{t_{j+1}}+\cdots+z_{l_{m}}^{t_{m}}
$$

then we have a decomposition with $\mathrm{h}(v)=s_{1}$ and $s_{1}>t_{j+1}>\cdots>t_{m}=s$ and $\mathrm{e}(v)=k>l_{j+1}>\cdots>l_{m}>0$.

It is not difficult to check that the following observation characterizes the numbers $m, k_{i}$ and $s_{i}$ in (1.5). For a nonzero element $x \in M$ with $\mathrm{e}(x)=k$ define $\mathrm{g}(x)=\mathrm{h}(x)+\mathrm{e}(x)$.

Lemma 2.3. Let $x \in M$ be decomposed as

$$
x=y_{k_{1}}^{s_{1}}+\cdots+y_{k_{m}}^{s_{m}}
$$

such that

$$
\mathrm{h}\left(y_{k_{i}}^{s_{i}}\right)=s_{i}, \mathrm{e}\left(y_{k_{i}}^{s_{i}}\right)=k_{i} \text {, and } y_{k_{i}}^{s_{i}} \text { is regular }, i=1, \ldots, m \text {. }
$$


and

$$
k_{1}>\cdots>k_{m}>0 \text { and } s_{1}>\cdots>s_{m} \text {. }
$$

Set $K=\left\{k_{1}, \ldots, k_{m}\right\}$. Then $j \in\{1, \ldots, k-1\}$, is in $K$ if and only if $g\left(p^{j} x\right)>g\left(p^{j-1} x\right)$. Moreover

$$
\mathrm{h}\left(p^{k_{j}-1} x\right)=\left(k_{j}-1\right)+s_{j}, j=1,2, \ldots m .
$$

In particular, we have $\mathrm{e}(x)=k_{1}$ and $\mathrm{h}(x)=s_{m}$.

\section{Equivalence of $(\mathrm{K})$ and $(\mathrm{B})$}

Condition $(\mathrm{K})$ can be reformulated in a more convenient form.

Lemma 3.1. We have

$$
p^{n} W \cap p^{n+r} M=p^{n}\left(W \cap p^{r} M\right), n \geq 0, r \geq 0,
$$

if and only if for each $w \in W$ with $\mathrm{h}\left(p^{n} w\right)=n+r$ there exists an element $\tilde{w} \in W$ such that

$$
p^{n} w=p^{n} \tilde{w} \text { and } \mathrm{h}(\tilde{w})=r .
$$

Proof: Obviously (3.1) is equivalent to

$$
p^{n} W \cap p^{n+r} M \subseteq p^{n}\left(W \cap p^{r} M\right), n \geq 0, r \geq 0 .
$$

Now (3.3) holds if and only if

$$
x \in p^{n} W, x \in p^{n+r} M \text { and } x \notin p^{n+r+1} M
$$

imply $x \in p^{n}\left(W \cap p^{r} M\right)$. That implication means the following. If $x=p^{n} w$ and $w \in W$ and $\mathrm{h}(x)=n+r$, then $x=p^{n} \tilde{w}$ for some $\tilde{w} \in W$ with $\mathrm{h}(\tilde{w}) \geq r$. Because of $\mathrm{h}\left(p^{n} \tilde{w}\right)=n+r$ the inequality $\mathrm{h}(\tilde{w}) \geq r$ is equivalent to $\mathrm{h}(\tilde{w})=r$.

Lemma 3.2. Let $x$ be an element of finite height with $\mathrm{e}(x)=k$. Then $x$ is regular if and only if the submodule $\langle x\rangle$ is regular, i.e.

$$
p^{r}\langle x\rangle \cap p^{n+r} M=p^{r}\left(\langle x\rangle \cap p^{n} M\right), n \geq 0, r \geq 0 .
$$


Proof: Assume (3.4). We want to show that $\mathrm{h}\left(p^{k-1} x\right)=(k-1)+s_{1}$ implies $s_{1}=\mathrm{h}(x)$. According to Lemma 3.1 there exists an element $\tilde{x} \in\langle x\rangle$ with properties corresponding to (3.2), i.e. $\tilde{x}=\gamma p^{t} x, \gamma \in R^{*}$, and $p^{k-1} x=$ $p^{k-1}\left(\gamma p^{t} x\right)$ and $\mathrm{h}\left(p^{t} x\right)=s_{1}$. Then we have $t=0$, and $\mathrm{h}(x)=s_{1}$. It is easy to check that that (3.4) holds if $x$ is regular .

Proof of Theorem 1.1, Part I: $(\mathrm{B}) \Leftrightarrow(\mathrm{K})$

$(\mathbf{B}) \Rightarrow(\mathbf{K})$ We want to show that condition $(\mathrm{B})$ implies $(\mathrm{K})$ in the equivalent form of Lemma 3.1. Let $w \in W$ be such that $\mathrm{h}\left(p^{n} w\right)=n+r$, and $h(w)=s$, $\mathrm{e}(w)=k_{1}$. Then $s \leq r$ and $k_{1}>n$. Hence $(\mathrm{B})$ yields a decomposition

$$
w=y_{k_{1}}^{s_{1}}+\cdots+y_{k_{m}}^{s_{m}}
$$

where the elements $y_{i}^{s_{i}} \in W$ are regular, $h\left(y_{i}^{s_{i}}\right)=s_{i}$, and

$$
s_{1}>\cdots>s_{m}=s=\mathrm{h}(w)
$$

and $\mathrm{e}(w)=k_{1}>\cdots>k_{m}>0$. Let $t$ be such that $k_{t}>n \geq k_{t+1}$. Then

$$
n+r=\mathrm{h}\left(p^{n} w\right)=\mathrm{h}\left(p^{n} y_{k_{1}}^{s_{1}}+\cdots+p^{n} y_{k_{t}}^{s_{t}}\right),
$$

and $\mathrm{h}\left(p^{n} w\right)=\mathrm{h}\left(p^{n} y_{k_{t}}^{s_{t}}\right)=n+s_{t}$. Hence $s_{t}=r$. Set $\tilde{w}=y_{k_{1}}^{s_{1}}+\cdots+y_{k_{t}}^{s_{t}}$. Then $\tilde{w} \in W$ and $\mathrm{h}(\tilde{w})=r$ and $p^{n} w=p^{n} \tilde{w}$.

$(\mathbf{K}) \Rightarrow(\mathbf{B})$ Because of Lemma2.2 it suffices to show that $(\mathrm{K})$ implies $(\mathrm{H})$. Let $x \in W$ be an $\left(s, k ; s_{1}\right)$-element. Set $w=p^{k-1} x$. Then $(\mathrm{K})$, resp. Lemma 3.1, imply that there exists an $\tilde{x} \in W$ such that

$$
p^{k-1} x=p^{k-1} \tilde{x}
$$

and $\mathrm{h}(\tilde{x})=s_{1}$. From (3.5) follows $\mathrm{e}(\tilde{x})=k$ and $\mathrm{h}\left(p^{k-1} \tilde{x}\right)=(k-1)+s_{1}$. Now set $z=x-\tilde{x}$. Then (3.5) yields $\mathrm{e}(z)<k$. Hence $x=\tilde{x}+z$ is a decomposition of type $(\mathrm{H})$.

As (K) holds for $W=M$ we can write each nonzero element $x$ of $M$ according to $(\mathrm{H})$ in the form (2.1). Similarly we can decompose $x$ according to (B) as a sum of the form (1.5). In that case we recover the result of Baer [4, p.4, Lemma 65.4] mentioned in Section 1.

\section{Equivalence of (B) and (FPP)}

In [2] J. Ferrer, and F. and X. Puerta studied marked invariant subspaces of an endomorphism $A$ of $\mathbb{C}^{n}$. Their investigation is based on subspaces of the 
form $\operatorname{Im}(\lambda I-A)^{s} \cap \operatorname{Ker}(\lambda I-A)^{k}$. Thus the submodules $M_{k}^{s}$ in (1.3) are a generalization of those subspaces. The next lemma is adapted from [2]. It characterizes regular elements in terms of $M_{k}^{s}$. Note that $M_{k}^{s} \subseteq M_{k_{1}}^{s_{1}}$ if $s_{1} \leq s$ and $k \leq k_{1}$. Hence $M_{k}^{s+1}+M_{k-1}^{s} \subseteq M_{k}^{s}$.

Lemma 4.1. An element $x \in M$ satisfies

$$
x \in M_{k}^{s} \text { and } x \notin M_{k}^{s+1}+M_{k-1}^{s}
$$

if and only if

$$
x \text { is regular and } \mathrm{h}(x)=s \text { and } \mathrm{e}(x)=k \text {. }
$$

Proof: " $\Rightarrow$ " Assume that $x$ satisfies (4.1). Recall that $x \in M_{k}^{s}$ if and only if both $\mathrm{h}(s) \geq s$ and $\mathrm{e}(x) \leq k$. Hence

$$
x \notin M_{k}^{s+1}+M_{k-1}^{s}
$$

implies $\mathrm{h}(x)=s$ and $\mathrm{e}(x)=k$. Assume $\mathrm{h}\left(p^{k-1} x\right)=(k-1)+s_{1}$. If we decompose $x$ according to $(\mathrm{H})$ then $x=y_{k}^{s_{1}}+z \in M_{k}^{s_{1}}+M_{k-1}^{s}$. Hence (4.3) implies $s_{1}=s$, and $x$ is regular.

" $\Leftarrow$ " Consider an element $x$ with properties (4.2). Then $x \in M_{k}^{s}, x \notin M_{k}^{s+1}$ and $x \notin M_{k-1}^{s}$. If

$$
x=y+z, y \neq 0, z \in M_{k-1}^{s},
$$

and $x$ is regular, then it follows from Lemma 2.1 that $\mathrm{h}(y)=s$. Hence we have $y \notin M_{k}^{s+1}$ and $x \notin M_{k}^{s+1}+M_{k-1}^{s}$.

Proof of Theorem 1.1, Part II: (H) $\Leftrightarrow$ (FPP)

$(\mathbf{H}) \Rightarrow(\mathbf{F P P})$ Because of the inclusion (1.8) the identity (1.7) in (FPP) is equivalent to

$$
W \cap\left(M_{k}^{s+1}+M_{k-1}^{s}\right) \subseteq\left(W \cap M_{k}^{s+1}\right)+\left(W \cap M_{k-1}^{s}\right) .
$$

We want to show that condition (H) implies (4.4) for all $s \geq 0, k \leq 1$. Take an element

$$
x \in W \cap\left(M_{k}^{s+1}+M_{k-1}^{s}\right) .
$$

Then $x \in M_{k}^{s}$ and therefore $\mathrm{h}(x) \geq s$ and $\mathrm{e}(x) \leq k$. To prove that

$$
x \in\left(W \cap M_{k}^{s+1}\right)+\left(W \cap M_{k-1}^{s}\right)
$$

we consider three cases. First, let $\mathrm{h}(x) \geq s+1$ then $x \in W \cap M_{k}^{s+1}$ and (4.6) is obvious. Secondly, let $\mathrm{e}(x) \leq k-1$. In that case $x \in W \cap M_{k-1}^{s}$. Now assume $\mathrm{h}(x)=s$ and $\mathrm{e}(x)=k$. By Lemma 4.1 it follows from (4.5) that $x$ is 
not regular. Hence $\mathrm{h}\left(p^{k-1} x\right)=(k-1)+s_{1}$ and $s_{1}>s$. According to $(\mathrm{H})$ we have $x=y_{k}^{s_{1}}+z$ with $y_{k}^{s_{1}} \in W \cap M_{k}^{s_{1}}$ and $z \in W \cap M_{k-1}^{s}$, which yields (4.6). $(\mathbf{F P P}) \Rightarrow(\mathbf{H})$ Let $x$ be an $\left(s, k ; s_{1}\right)$-element. If $s_{1}=s$ then $x$ is regular and we have (2.1) with $z=0$. Suppose now that $x$ is not regular, i.e. $s_{1} \geq s+1$. Then Lemma 4.1 implies $x \in W \cap\left(M_{k}^{s+1}+M_{k-1}^{s}\right)$. From (FPP) we obtain

$$
x=y+z, y \in W \cap M_{k}^{s+1}, z \in W \cap M_{k-1}^{s} .
$$

Then $y \neq 0, \mathrm{e}(y)=k$ and $\mathrm{h}(y) \geq s+1$. Let $y$ in (4.7) be such that $\mathrm{h}(y)$ is maximal. We shall see that such a choice of $y$ implies $\mathrm{h}(y)=s_{1}$, and in that case (4.7) is a decomposition of type $(\mathrm{H})$. Now suppose that $\mathrm{h}(y)=\tilde{s}<s_{1}$. Then, by Lemma 2.1, the element $y \in W$ is not regular. Applying Lemma 4.1 to $y \in W \cap M_{k}^{\tilde{s}}$ we obtain $y \in W \cap\left(M_{k}^{\tilde{s}+1}+M_{k-1}^{\tilde{s}}\right)$. Thus (FPP) yields

$$
y=\tilde{y}+z_{2}, \tilde{y} \in W \cap M_{k}^{\tilde{s}+1}, \tilde{y} \neq 0, z_{2} \in W \cap M_{k-1}^{\tilde{s}} .
$$

Hence $x=\tilde{y}+\left(z+z_{2}\right)$, and we have another decomposition of the form (4.7), but now with $h(\tilde{y})>\tilde{s}$, which contradics the maximality of $\tilde{s}$.

Acknowledgement. We would like to thank O. Mutzbauer for a valuable comment.

\section{References}

[1] Baer, R.: Types of elements and the characteristic subgroups of abelian groups. Proc. London Math. Soc. 39(1935), 481-514.

[2] Ferrer, J.; Puerta, F.; Puerta, X.: Geometric characterization and classification of marked subspaces. Linear Algebra Appl. 235(1996), 15-34.

[3] Fuchs, L.: Infinite Abelian Groups, Vol. I. Academic Press, New York 1973.

[4] Fuchs, L.: Infinite Abelian Groups, Vol. II. Academic Press, New York 1973.

[5] Kaplanski, I.: Infinite Abelian Groups. University of Michigan Press, Ann Arbor 1954.

[6] Vilenkin, N. Ya.: Direct decompositions of topological groups, I. (in Russian). Mat. Sbornik N. S. 19(1946), 85-154. 